\title{
Study reveals secrets to faster drug development
}

Effective and efficient decision-making is still a major roadblock in companies

\section{Simon Frantz}

For a business in which ultimate success — the approval of new drugs - is achieved over a time frame of years and decades, it might seem odd to look at ways in which companies can shave mere days off the development process.

But bringing a new drug to market involves a vast array of disciplines, and minor delays within individual disciplines soon add up and increase development costs for companies, not to mention losing valuable patent exclusivity time. With the average blockbuster drug bringing in around US\$1 million a day in revenues, wasted days really can hit companies' pockets hard.

So a great deal of effort is spent trying to identify where time improvements can be made without sacrificing quality. The latest contribution, a report from the Tufts Center for the Study of Drug Development, names the fastest drug development companies, and proposes the secret to their success.

Over the development and regulatory period for drugs approved by the FDA between 1994-2005, Bayer, AstraZeneca, Allergan, Boehringer Ingelheim and Merck were the fastest development companies, or, as the study authors call them, 'speed demons' (Tufts CSDD Impact Report Sept/Oct 2006). These five companies, according to the report "deliver as much as a 17-month speed advantage over average performers".

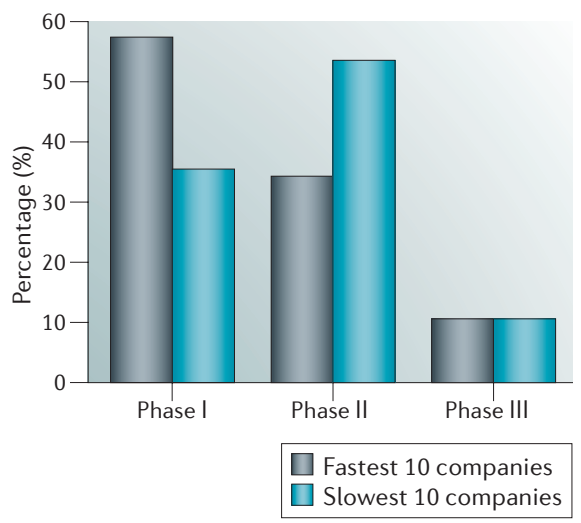

Figure 1 | Faster companies kill projects sooner than slower firms. Source: Tufts CSDD.
It is always difficult to apply these data across the board because of the development and regulatory nuances within individual therapeutic areas. But interviews with clinical development executives at the speed-demon companies showed there were several factors that set them apart from slower drug developers. "There is no single way to be the fastest; it is all about the commitment to consistently apply strategies and practices across the portfolio," says Kenneth Getz, senior research fellow at the Tufts Center, and lead author of the study.

Executives at the speed-demon companies cited four main areas that made them efficient. Three areas point to external influences: the enterprise-wide adoption of e-clinical technology solutions; the high usage

\section{Making efficient and effective decisions between phases is still one of the major impasses in developing a drug}

of contract clinical service providers; and active interaction with regulatory agencies. The fourth area, though, is one that strikes at the very heart of a company's make-up: effective management and prioritization of resources, including the termination of poor projects sooner. And making efficient and effective decisions between phases is still one of the major impasses in developing a drug, say analysts.

Companies have worked hard to make project phases more efficient, but they still need to improve on the so-called 'frictional' times and costs incurred by delaying decisions to move from one phase to another. "Somebody has to declare that the trial is stopped, somebody has to make a decision about that trial, and somebody has to get the process going for the next set of trials," says Navjot Singh,

Associate Principal at McKinsey and Company. "All of that takes weeks, if not months, and the question is how can you save on that?"

Part of the problem lies with the decisionmaking structure in pharmaceutical companies. Decisions still tend to be made at the highest possible level, which means decision-makers have less insight into what people are working on. Project teams go to the decision-makers fighting for their compounds as much as possible, and are reluctant to give up on their project, in the hope that a project will succeed through perseverance. This makes it easy for projects to run beyond the point where the team knows they are not going to work. One major consequence of ineffective management is illustrated in the Tufts report. The fastest companies terminated $56 \%$ of discontinued projects in Phase I, compared with $36 \%$ for slowest companies, and the situation is almost reversed for Phase II (FIG. 1).

Another problem is that decisions to go forward on projects often lay stagnant within the layers of middle management. Efficient companies are more likely to have leaner and meaner decision-making structures, and a greater focus of competencies, says Nipon Das, President \& Managing Director of Billinge Group, LLC. These companies have built strong competencies and incorporate their experience and talent to make quick decisions. Larger companies can become inefficient by trying to manage too many portfolios. "I am always concerned with companies spread across too many therapeutic areas,' says Das.

With the exception of AstraZeneca, the five named companies in the report have grown via a culture of organic growth (although AstraZeneca has been integrated for many years). "I have seen the after-effects of sequential merger and acquisition growth: the loss of good people with experience and decision-making skills," says Das. "You cannot put too high a premium on the people that helped get an acquired product to market in the first place."

Singh agrees that more knowledge input is needed from people closer to the bench.

"These people often know whether something is working or not," he says. "If you can distribute the ownership of resource management effectively at lower levels of management, and with the right incentives, these people have experience and information that can help you make a decision." 